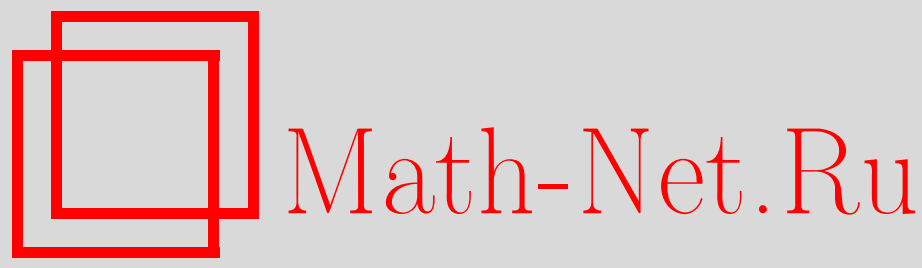

В. Г. Журавлев, Многогранники ограниченного остатка, Совр. пробл. матем., 2012, выпуск 16, 82-102

DOI: https://doi.org/10.4213/spm37

Использование Общероссийского математического портала Math-Net.Ru подразумевает, что вы прочитали и согласны с пользовательским соглашением http://www . mathnet.ru/rus/agreement

Параметры загрузки:

IP : 54.224 .60 .19

26 апреля 2023 г., 06:02:39

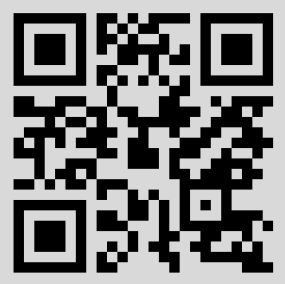




\title{
Многогранники ограниченного остатка
}

\author{
В. Г. Журавлёв \\ Владимирский государственный университет
}

Последнему рыцарю теории чисел посвящается

\section{Введение}

0.1. Одномерный случай. Гекке в 1921 г. доказал теорему [1]: для отклонений $\delta(i, X)=$ $r(i, X)-i|X|$, где $r(i, X)$ - количество точек орбиты длинь $i$ относительно вращения $S_{\alpha}(x) \equiv x+\alpha \bmod 1$ окружности единичной длины на угол $\alpha$, попавших в отрезок $X$, выполняется неравенство

$$
|\delta(i, X)| \leqslant h
$$

при условии, что отрезок $X$ имеет длину $|X|=h \alpha+b$, где $h \in \mathbb{N}, b \in \mathbb{Z}$.

Орен [2] смог заменить отрезок в теореме Гекке конечным объединением интервалов $X$ и получил для таких множеств оценку

$$
\delta(i, X)=O(1)
$$

при этом отдельные интервалы из множества $X$ сами могут и не обладать свойством $(0.2)$. Если выполняется условие (0.2), то $X$ называется множеством ограниченного остатка или, кратко, BR-множеством.

0.2. Многомерный случай. В 1954 г. Сюс [3] построил первое семейство параллелограммов $X^{2} \subset \mathbb{T}^{2}$, для которых выполняется оценка $(0.2)$. Анализ конструкции Сюс привел Лиарде [4] к открытию возможной редукции от $B R$-множеств $X^{D}$ к аналогичным множествам меньшей размерности $X^{D-1}$. Это был важный шаг, так как он позволял строить $B R$-множества $X^{D}$ для произвольной размерности $D$. Так, например, параллелограммы Сюса $X^{2}$ получаются подъемом отрезков Гекке $X^{1}$.

Неожиданный подход к построению множеств $X^{D}$ обнаружили Рози [5] и Ференци [6]. Они связали свойство $X^{D}$ быть $B R$-множеством со свойствами соответствующего индуцированного отображения $S_{\alpha} \mid X^{D}$ - отображения первого возвращения или отображения Пуанкаре. Идея Рози-Ференци была реализована в [7], где доказано общее неравенство

$$
\left|\delta\left(i, X^{2}\right)\right|<2
$$

для всех множеств $X^{2} \subset \mathbb{T}^{2}$ из последовательности самоподобных фрактальных областей Рози $\mathcal{R}_{1} \supset \cdots \supset \mathcal{R}_{h} \supset \cdots$, обладающих свойством $\bigcap_{h=1}^{\infty} \mathcal{R}_{h}=\{0\}$.

Глобальный подход к поиску $B R$-множеств был предложен в [8], где вместо отдельных множеств $X_{k}^{D} \subset \mathbb{T}^{D}$ стали рассматриваться полные разбиения торов $\mathbb{T}_{c, \lambda}^{D}=X_{0}^{D} \sqcup X_{1}^{D} \sqcup \cdots \sqcup X_{s}^{D}$ с некоторыми параметрами $c, \lambda$. Основная идея состояла в том, чтобы определить подъем тора $\mathbb{T}^{D}$ в накрывающее пространство $\mathbb{R}^{D}$ :

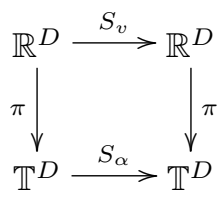

Работа выполнена при финансовой поддержке Российского фонда фундаментальных исследований (грант № 11-01-00578-a). 
при котором повороту тора $S_{\alpha}$ отвечает перекладывание $S_{v}$ некоторых множеств $X_{0}^{\prime}, X_{1}^{\prime}, \ldots$, $X_{s}^{\prime}$ из $\mathbb{R}^{D}$. Если число таких множеств $X_{k}^{\prime}$ окажется $s+1 \leqslant D+1$, то каждый из образов $X_{k}^{D}=\pi\left(X_{k}^{\prime}\right)$ на торе $\mathbb{T}^{D}$ будет $B R$-множеством, а соответствующее объединение $T_{c, \lambda}^{D}=X_{0}^{\prime} \sqcup$ $X_{1}^{\prime} \sqcup \cdots \sqcup X_{s}^{\prime}$ из $\mathbb{R}^{D}$ - перекладывающейся торической разверткой $T^{D}=\pi^{-1}\left(\mathbb{T}^{D}\right) \subset \mathbb{R}^{D}$ для $\mathbb{T}^{D}$. Такие развертки $T^{D}$ были сконструированы в [9] с помощью операции вытягивания единичных кубов.

0.3. $\boldsymbol{B R}$-многогранники. Цель настоящей работы - на основе схемы (0.4) представить систематический подход [8]-[10] к построению многомерных $B R$-множеств. Мы останавливаемся исключительно на выпуклых $B R$-множествах, хотя методы [9] позволяют исследовать и невыпуклый случай.

Основными являются теоремы 4.1 и 5.2, в которых для произвольной размерности $D$ строятся $B R$-многогранники $X^{D}=P_{k}$ : параллелепипеды и выпуклые параллелоэдры с числом вершин $\# V\left(P_{k}\right)=2^{D+1}-2$. Для размерностей $D=1$ и 2 это будут множества, содержащие соответственно отрезки и шестиугольники с попарно параллельными и равными сторонами, а для $D=3,4$ - параллелоэдры Вороного [11], среди которых содержится, например, ромбический додекаэдр. Для указанных $B R$-многогранников $X^{D}=P_{k}$ в теореме 5.2 доказывается неравенство

$$
\left|\delta\left(i, X^{D}\right)\right| \leqslant D h
$$

- многомерный аналог теоремы Гекке (0.1) и неравенства (0.3). В неравенстве (0.5) отклонения $\delta\left(i, X^{D}\right)$ рассматриваются для сдвига тора $S_{\beta}$ на вектор $\beta=(\alpha+l) / h$, где $l$ - произвольный вектор из кубической решетки $\mathbb{Z}^{D}$.

\section{1. Торические развертки и множества ограниченного остатка}

1.1. Перекладывающиеся торические развертки. Пусть дан $D$-мерный тор

$$
\mathbb{T}^{D} \simeq \mathbb{R}^{D} / L
$$

где $L$ - невырожденная решетка из вещественного арифметического векторного пространства $\mathbb{R}^{D}$, т.е. решетка $L$ имеет размерность $D$ над $\mathbb{R}$. Пусть задан сдвиг тора

$$
\mathbb{T}^{D} \stackrel{S_{\alpha}}{\longrightarrow} \mathbb{T}^{D}: \quad x \mapsto S_{\alpha}(x) \equiv x+\alpha \bmod L .
$$

Разверткой $T^{D}$ тора $\mathbb{T}^{D}$ назовем такое подмножество $T^{D}$ из $\mathbb{R}^{D}$, что ограничение фактор отображения

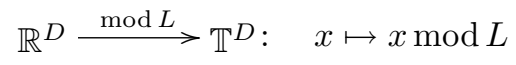

на указанное подмножество $T^{D} \subset \mathbb{R}^{D}$ задает биекцию

$$
T^{D \stackrel{\bmod L}{\longrightarrow}} \mathbb{T}^{D}
$$

Пусть дана торическая развертка $T^{D}$, удовлетворяющяя следующему условию. Существует такое разбиение

$$
T^{D}=T_{0} \sqcup T_{1} \sqcup \cdots \sqcup T_{D}
$$

развертки $T^{D}$, что если с помощью биекции (1.3) отождествить тор $\mathbb{T}^{D}$ с его разверткой $T^{D}$, то индуцированное отображение $T^{D} \stackrel{S_{v}}{\longrightarrow} T^{D}$ для сдвига тора (1.2) будет эквивалентно перекладыванию областей $T_{0}, T_{1}, \ldots, T_{D}$ развертки $T^{D}$ :

$$
S_{v}(x)=x+v(x),
$$


где вектор сдвига $v(x)$ зависит от $x \in T^{D}$ и определяется условием

$$
v(x)=v_{k}, \quad \text { если } \quad x \in T_{k},
$$

при этом $v_{0}, v_{1}, \ldots, v_{D}$ - некоторая фиксированная система векторов.

Заметим, что разбиению (1.4) развертки $T^{D}$ отвечает разбиение

$$
\mathbb{T}^{D}=\mathbb{T}_{0} \sqcup \mathbb{T}_{1} \sqcup \cdots \sqcup \mathbb{T}_{D}
$$

тора $\mathbb{T}^{D}$ на области $\mathbb{T}_{k} \equiv T_{k} \bmod L$.

\section{2. Примеры перекладывающихся торических разверток.}

1.2.1. Проекции куба. Обозначим через $C^{D+1}$ единичный $(D+1)$-мерный куб, вложенный в пространство $\mathbb{R}^{D+1}$. Его проекцией на подпространство $\mathbb{R}^{D} \subset \mathbb{R}^{D+1}$ вдоль некоторого направления $\mathbf{p} \in \mathbb{R}^{D+1}$ будет $D$-мерный многогранник $\mathcal{P}^{D}$. Выбранное направление $\mathbf{p}$ условно разделяет $D$-мерные грани куба $C^{D+1}$ на два вида: видимые и невидимые.

Так, например, в случае $D=1$ стороны квадрата $C^{2}$ при проектировании на прямую вдоль вектора общего положения р разделяются на пары видимых и невидимых, для $D=2$ шесть граней куба $C^{3}$ при проектировании на плоскость также распадаются на два множества, состоящие соответственно из трех видимых и трех невидимых граней.

Возвращаясь к произвольной размерности $D$ и проектируя, скажем, первые грани, получим

$$
\mathcal{P}^{D}=\mathcal{P}_{0} \cup \mathcal{P}_{1} \cup \cdots \cup \mathcal{P}_{D}
$$

- разбиение $\mathcal{P}^{D}$ на многогранники $\mathcal{P}_{k}$ без общих внутренних точек. Заметим, что в вырожденном случае количество многогранников в (1.8) может быть меньше $D+1$. Если же затем спроектировать грани второго вида, то снова придем к разбиению (1.8), в котором многогранники $\mathcal{P}_{k}$ некоторым образом переложены параллельными сдвигами. Это явление объясняется тем, что для каждой $D$-грани куба $C^{D+1}$ существует параллельная ей $D$-грань.

Многогранник $\mathcal{P}^{D}$ является параллелоэдром: он разбивает пространство $\mathbb{R}^{D}$ на области $\mathcal{P}^{D}[l]=\mathcal{P}^{D}+l$ параллельными сдвигами на векторы $l$ из некоторой решетки $L$ :

$$
\mathbb{R}^{D}=\coprod_{l \in L} \mathcal{P}^{D}[l]
$$

Поэтому после отбрасывания лишних граничных точек $\mathcal{P}^{D}$ превращается в развертку тора $\mathbb{T}^{D} \simeq \mathbb{R}^{D} / L$. При этом можно проверить, что перекладыванию многогранников $\mathcal{P}_{k}$ из разбиения (1.8) соответствует некоторый сдвиг тора $\mathbb{T}^{D}$, и, таким образом, мы получаем перекладывающуюся торическую развертку $\mathcal{P}^{D}$ (детали см. в п. 3.4).

Так, в случае $D=1$ возникает отрезок, составленный из двух ме́ньших отрезков, а при $D=$ 2 получается шестиугольник $\mathcal{P}^{D}$ с попарно параллельными и равными сторонами, разбитый на три перекладывающихся параллелограмма.

В разделах 3, 4 мы рассмотрим приведенную выше конструкцию и даже более общую конструкцию, комбинируя проекцию $\mathbf{p}$ с некоторой дополнительной операцией вытягивания кубов.

1.2.2. Произведения торических разверток. В [9] были определены некоммутативные произведения $T^{D_{1}} \otimes_{i} T^{D_{2}}$, позволяющие из двух торических разверток $T^{D_{1}}$ и $T^{D_{2}}$ строить новую развертку тора $\mathbb{T}^{D}$ размерности $D=D_{1}+D_{2}$. Операция умножения обладает свойством: если исходные развертки $T^{D_{1}}$ и $T^{D_{2}}$ были перекладывающимися, то любое их произведение $T^{D_{1}} \otimes_{i} T^{D_{2}}$ также будет перекладьвающейся торической разверткой.

Например, если выбрать в качестве разверток $T^{D_{1}}, T^{D_{2}}$ перекладывающиеся отрезки, то их произведения $T^{D_{1}} \otimes_{i} T^{D_{2}}$ представляют собой прямоугольники, разбитые на три перекладывающиеся области, каждая из которых также является некоторым прямоугольником. В частности, на этом пути после подходящего гиперболического автоморфизма тора получаются параллелограммы Сюс [3]. 
1.3. Трансляционная решетка и ранг вектора сдвига. Из определений (1.2), (1.5) и (1.6) вытекают сравнения

$$
v_{k} \equiv \alpha \bmod L \quad \text { для } \quad k=0,1, \ldots, D,
$$

при этом векторы

$$
l_{k}=v_{k}-v_{0} \quad \text { для } \quad k=1, \ldots, D
$$

принадлежат основной решетке периодов $L$ тора (1.1). В дальнейшем на протяжении всей статьи будем предполагать, что $L$ является невырожденной решеткой, порождаемой

$$
L=\mathbb{Z}\left[l_{1}, \ldots, l_{D}\right]
$$

векторами (1.10) над кольцом целых чисел $\mathbb{Z}$, т.е.

$$
\text { система векторов } l_{1}, \ldots, l_{D} \text { имеет ранг } D \text { над } \mathbb{R} \text {. }
$$

Следовательно, при этом условии вектор сдвига $\alpha$ можно разложить по данному базису:

$$
\alpha=\alpha_{1} l_{1}+\cdots+\alpha_{D} l_{D} .
$$

Пусть $\alpha$ - произвольный вектор из $\mathbb{R}^{D}$ с координатами $\left(\alpha_{1}, \ldots, \alpha_{D}\right)$ в базисе $(1.13)$. Определим его ранг относителъно решетки $L$ равенством

$$
\operatorname{rank}_{L}(\alpha)=\operatorname{rank}_{\mathbb{Z}} M(\alpha)-1,
$$

где $M(\alpha)=\mathbb{Z}\left[1, \alpha_{1}, \ldots, \alpha_{D}\right] \subset \mathbb{R}$ обозначает модуль над кольцом $\mathbb{Z}$, порождаемый числами $1, \alpha_{1}, \ldots, \alpha_{D} ; \operatorname{rank}_{\mathbb{Z}} \mathbb{Z}\left[1, \alpha_{1}, \ldots, \alpha_{D}\right]$ - размерность этого модуля над $\mathbb{Z}$. Если $\alpha^{\prime} \subset \mathbb{R}^{D}-$ другой вектор, удовлетворяющий условию $\alpha^{\prime} \equiv \alpha \bmod L$, то из определения (1.14) вытекает

$$
\operatorname{rank}_{L}\left(\alpha^{\prime}\right)=\operatorname{rank}_{L}(\alpha)
$$

Это означает, что ранг $\operatorname{rank}_{L}(\alpha)$ корректно определен для векторов $\alpha$ на торе $\mathbb{T}^{D} \simeq \mathbb{R}^{D} / L$. Из определения (1.14) также следуют неравенства

$$
0 \leqslant \operatorname{rank}_{\mathbb{Z}}(\alpha) \leqslant D
$$

Назовем вектор $\alpha$ иррациональным, если $\operatorname{rank}_{\mathbb{Z}}(\alpha)>0$. Если при этом ранг принимает наибольшее значение $\operatorname{rank}_{\mathbb{Z}}(\alpha)=D$, то будем говорить, что вектор максимально иррациональный. В оставшемся случае $\operatorname{rank}_{\mathbb{Z}}(\alpha)=0$ скажем, что вектор рационалъный. Такое название объяняется тем, что координаны $\alpha_{1}, \ldots, \alpha_{D}$ в разложении (1.13) - рациональные числа.

Для вектора $\alpha$ отвечающие ему сдвиг $S_{\alpha}$ тора (1.2) и перекладывание $S_{v}$ из (1.6) будем называть соответственно иррациональными, максимально иррациональными и рациональнымu.

1.4. Векторная $\boldsymbol{T}$-дробная часть. Для любого $x \in \mathbb{R}^{D}$ определим его векторную дробную часть $\operatorname{Fr}(x)$, полагая

$$
\operatorname{Fr}(x)=\bar{x}
$$

где $\bar{x} \equiv x \bmod L$ и $\bar{x}$ принадлежит развертке $T=T^{D} \subset \mathbb{R}^{D}$. Корректность определния (1.15) вытекает из факта существования разбиения

$$
\mathbb{R}^{D}=\coprod_{l \in L} T[l]
$$

пространства $\mathbb{R}^{D}$ на области $T[l]=T+l=\{x+l: x \in T\}$, получающиеся параллельным сдвигом области $T$ на векторы $l$ из решетки $L$. Чтобы указать зависимость дробной части $\operatorname{Fr}(x)$ 
от развертки $T=T^{D}$, мы будем также использовать дополнительное обозначение $\operatorname{Fr}_{T}(x)$, и точку или вектор $\operatorname{Fr}_{T}(x)$ будем называть $T$-дробной частью $x \in \mathbb{R}^{D}$.

Пусть

$$
\Delta \operatorname{Fr}(x)=\operatorname{Fr}(x+\alpha)-\operatorname{Fr}(x)
$$

- векторно значная разностная функция с шагом $\alpha$, где $\alpha$ - вектор сдвига (1.2) тора $\mathbb{T}^{D}$. Тогда выполняется следующая формула [8]

$$
\Delta \operatorname{Fr}(x)=v(\bar{x})
$$

для любого $x \in \mathbb{R}^{D}$, где $\bar{x}=\operatorname{Fr}(x)$ по определению $T$-дробной части (1.15) принадлежит развертке $T$ и вектор $v(\bar{x})$ согласно (1.5) представим в виде суммы

$$
v(\bar{x})=\alpha+l(\bar{x}),
$$

при этом $l(\bar{x})=l_{k}$, если $\bar{x} \in T_{k}$ для $k=1, \ldots, D$, и $l(\bar{x})=l_{0}=0$, если $\bar{x} \in T_{0}$. Здесь $l_{k}-$ векторы (1.10).

1.5. Суммарное векторное отклонение. Для $i=0,1,2, \ldots$ определим векторно значную функцию $\delta\left(i, x_{0}\right)$, полагая

$$
\delta\left(i, x_{0}\right)=\sum_{0 \leqslant j<i} \Delta \operatorname{Fr}\left(j \beta+x_{0}\right)
$$

где

$$
\beta=\frac{1}{h}(\alpha+l),
$$

при этом $h$ - произвольное натуральное число и $l$ - любой вектор из решетки $L$. Используя формулу (1.17), сумму (1.19) можем преобразовать к следующему виду:

$$
\delta\left(i, x_{0}\right)=\sum_{0 \leqslant j<i} v\left(\bar{x}_{j}\right)
$$

Здесь $x_{j}=j \beta+x_{0}$ и все точки $\bar{x}_{j}=\operatorname{Fr}\left(x_{j}\right)$ принадлежат развертке тора $T$. Теперь, поставляя в сумму (1.21) выражение (1.18) для вектора $v(\bar{x})$, можем записать

$$
\delta\left(i, x_{0}\right)=\sum_{0 \leqslant j<i}\left(\alpha+l\left(\bar{x}_{j}\right)\right)=i \alpha+\sum_{0 \leqslant j<i} l\left(\bar{x}_{j}\right)=i \alpha+\sum_{\substack{0 \leqslant k \leqslant D \\ 0 \leqslant j<i \\ 0, \bar{x}_{j} \in T_{k}}} l\left(\bar{x}_{j}\right) .
$$

Так как $l\left(\bar{x}_{j}\right)=l_{k}$, если $\bar{x}_{j} \in T_{k}$, и $l_{0}=0$ по определению $(1.18)$, то из $(1.22)$ для $\delta\left(i, x_{0}\right)$ вытекает представление

$$
\delta\left(i, x_{0}\right)=i \alpha+\sum_{0 \leqslant k \leqslant D} l_{k} \sum_{\substack{0 \leqslant j<i \\ \operatorname{Fr}\left(j \beta+x_{0}\right) \in T_{k}}} 1
$$

или, в другой форме,

$$
\delta\left(i, x_{0}\right)=i \alpha+r_{1}\left(i, x_{0}\right) l_{1}+\cdots+r_{D}\left(i, x_{0}\right) l_{D}
$$

где

$$
r_{k}\left(i, x_{0}\right)=\#\left\{\operatorname{Fr}\left(j \beta+x_{0}\right) \in T_{k}: 0 \leqslant j<i\right\} .
$$

Пусть $\mathbb{T}^{D}=\mathbb{T}_{0} \sqcup \mathbb{T}_{1} \sqcup \cdots \sqcup \mathbb{T}_{D}$ - разбиение тора (1.7), отвечающее разбиению (1.4) развертки тора $T^{D}$. Если теперь воспользоваться сдвигом тора

$$
\mathbb{T}^{D} \stackrel{S_{\beta}}{\longrightarrow} \mathbb{T}^{D}: \quad x \mapsto S_{\beta}(x) \equiv x+\beta \bmod L
$$


на вектор $\beta$, определенный равенством (1.20), то из сравнения

$$
\operatorname{Fr}\left(j \beta+x_{0}\right) \equiv S_{\beta}^{j}\left(x_{0}\right) \bmod L
$$

следует, что считающую функцию (1.24) можно записать в виде

$$
r_{k}\left(i, x_{0}\right)=\#\left\{j: S_{\beta}^{j}\left(x_{0}\right) \in \mathbb{T}_{k}, 0 \leqslant j<i\right\} .
$$

Сумму (1.19) можно также вычислить иным способом. Используя определение (1.19), запишем $\delta\left(i, x_{0}\right)$ в виде разности сумм:

$$
\delta\left(i, x_{0}\right)=\sum_{0 \leqslant j<i} \operatorname{Fr}\left(j \beta+\alpha+x_{0}\right)-\sum_{0 \leqslant j<i} \operatorname{Fr}\left(j \beta+x_{0}\right) .
$$

Согласно (1.20) имеем

$$
\alpha \equiv h \beta \bmod L .
$$

Тогда из (1.28) и определения (1.16) векторной $T$-дробной части $\operatorname{Fr}(x)$ следует равенство

$$
\operatorname{Fr}\left(j \beta+\alpha+x_{0}\right)=\operatorname{Fr}\left(j \beta+h \beta+x_{0}\right),
$$

из которого вытекает

$$
\sum_{0 \leqslant j<i} \operatorname{Fr}\left(j \beta+\alpha+x_{0}\right)=\sum_{0 \leqslant j<i} \operatorname{Fr}\left(j \beta+h \beta+x_{0}\right)=\sum_{h \leqslant j<i+h} \operatorname{Fr}\left(j \beta+x_{0}\right) .
$$

Поэтому из $(1.27)$ и $(1.29)$ для функции $\delta\left(i, x_{0}\right)$ получаем еще одно представление

$$
\delta\left(i, x_{0}\right)=\sum_{h \leqslant j<i+h} \operatorname{Fr}\left(j \beta+x_{0}\right)-\sum_{0 \leqslant j<i} \operatorname{Fr}\left(j \beta+x_{0}\right) .
$$

Предположим, что $i>h$. Тогда из (1.30) вытекают равенства

$$
\begin{aligned}
\delta\left(i, x_{0}\right) & =\sum_{h \leqslant j<i+h} \operatorname{Fr}\left(j \beta+x_{0}\right)-\sum_{0 \leqslant j<i} \operatorname{Fr}\left(j \beta+x_{0}\right) \\
& =\sum_{0 \leqslant j \leqslant h-1}\left(\operatorname{Fr}\left(j \beta+i \beta+x_{0}\right)-\operatorname{Fr}\left(j \beta+x_{0}\right)\right) .
\end{aligned}
$$

ПрЕДЛОЖЕНИЕ 1.1. 1) Пусть вектор сдвига $\beta$ определен равенством $(1.20)$ и $\delta\left(i, x_{0}\right)$ векторно-значная функция (1.19). Тогда $\delta\left(i, x_{0}\right)$ представима в виде суммь

$$
\delta\left(i, x_{0}\right)=i \alpha+r_{1}\left(i, x_{0}\right) l_{1}+\cdots+r_{D}\left(i, x_{0}\right) l_{D},
$$

где $r_{k}\left(i, x_{0}\right)$ равно количеству попаданий начальной точки $x_{0}$ под действием сдвига $S_{\beta}$ в область $\mathbb{T}_{k}$ из рабиения (1.7) тора $\mathbb{T}^{D}$. Кроме (1.32) вектор функиия $\delta\left(i, x_{0}\right)$ может быть представлена еще в одном из двух видов:

$$
\delta\left(i, x_{0}\right)=\sum_{0 \leqslant j<i}\left(\operatorname{Fr}\left(j \beta+h \beta+x_{0}\right)-\operatorname{Fr}\left(j \beta+x_{0}\right)\right)
$$

$\partial л я 0 \leqslant i \leqslant h u$

$$
\delta\left(i, x_{0}\right)=\sum_{0 \leqslant j \leqslant h-1}\left(\operatorname{Fr}\left(j \beta+i \beta+x_{0}\right)-\operatorname{Fr}\left(j \beta+x_{0}\right)\right)
$$

для $i>h$. Здесъ $\mathrm{Fr}=\operatorname{Fr}_{T}(x)$ определено равенством (1.15) и равно векторной T-дробной части точки $x \in \mathbb{R}^{D}$.

2) В частности, если $h=1$, то в этом случае вектором сдвига будет $\beta=\alpha$ и формуль (1.33) и (1.34) принимают единообразный вид

$$
\delta\left(i, x_{0}\right)=\operatorname{Fr}\left(i \alpha+x_{0}\right)-\operatorname{Fr}\left(x_{0}\right)
$$

для любого $i=0,1,2, \ldots$. 
ДоказАтельство. Формула (1.32) была доказана в (1.23). Равенство (1.33) вытекает непосредственно из определения (1.19) и сравнения (1.28), а равенство (1.34) совпадает с (1.31).

1.6. Цветные $\boldsymbol{k}$-отклонения. По условию $L$ - невырожденная решетка (1.11). Поэтому для ее базиса $l_{1}, \ldots, l_{D}$ существует двойственный базис $l_{1}^{*}, \ldots, l_{D}^{*}$, связанный с исходным базисом соотношениями

$$
l_{k}^{*} \cdot l_{m}=\delta_{k m}
$$

где $x \cdot y=x_{1} y_{1}+\cdots+x_{D} y_{D}-$ скалярное произведение и $\delta_{k m}-$ символ Кронекера. Используя двойственный базис (1.36) и представление (1.32), получаем следующие равенства

$$
l_{k}^{*} \cdot \delta\left(i, x_{0}\right)=r_{k}\left(i, x_{0}\right)+i l_{k}^{*} \cdot \alpha \quad \text { для } \quad k=1, \ldots, D .
$$

Обозначим

$$
\delta_{k}\left(i, x_{0}\right)=l_{k}^{*} \cdot \delta\left(i, x_{0}\right)
$$

и перепишем (1.37) в виде

$$
\delta_{k}\left(i, x_{0}\right)=r_{k}\left(i, x_{0}\right)-i a_{k} \quad \text { для } \quad k=1, \ldots, D .
$$

Здесь коэффициенты $a_{k}$ задаются равенством

$$
a_{k}=-l_{k}^{*} \cdot \alpha=-\alpha_{k},
$$

где $\alpha_{k}$ - координаты (1.13) вектора $\alpha$ относительно базиса $l_{1}, \ldots, l_{D}$.

Назовем $\delta_{k}\left(i, x_{0}\right)$ из (1.39) отклонением распределения точек орбиты

$$
\operatorname{Orb}_{S_{\beta}}\left(x_{0}\right)=\left\{S_{\beta}^{i}\left(x_{0}\right) \equiv x_{0}+i \beta \bmod L, i=0,1,2, \ldots\right\}
$$

относительно области $\mathbb{T}_{k} \subset \mathbb{T}^{D}$ либо, кратко, $k$-отклонением или иветным отклонением.

Из (1.37) и (1.39) следует, что вектор функция $\delta\left(i, x_{0}\right)$ и цветные отклонения $\delta_{k}\left(i, x_{0}\right)$ связаны между собой соотношением

$$
\delta\left(i, x_{0}\right)=\delta_{1}\left(i, x_{0}\right) l_{1}+\cdots+\delta_{D}\left(i, x_{0}\right) l_{D} .
$$

Поэтому $\delta\left(i, x_{0}\right)$ естественно назвать векторным суммарным отклонением распределения точек орбиты $\operatorname{Orb}_{S_{\beta}}\left(x_{0}\right)$ относительно всех областей $\mathbb{T}_{1}, \ldots, \mathbb{T}_{D}$ из разбиения (1.7) тора $\mathbb{T}^{D}$.

Остается выяснить вопрос о вычислении отклонения $\delta_{0}\left(i, x_{0}\right)$ относительно области $\mathbb{T}_{0} \subset$ $\mathbb{T}^{D}$. Заметим, что считающие функции $r_{0}\left(i, x_{0}\right), r_{1}\left(i, x_{0}\right), \ldots, r_{D}\left(i, x_{0}\right)$ не являются независимыми, так как непосредственно из определений (1.24) и (1.26) следует, что они удовлетворяют тождеству

$$
r_{0}\left(i, x_{0}\right)+r_{1}\left(i, x_{0}\right)+\cdots+r_{D}\left(i, x_{0}\right)=i
$$

для любого $i=0,1,2, \ldots$. Определим $a_{0}$ равенством

$$
a_{0}+a_{1}+\cdots+a_{D}=1 .
$$

Согласно (1.43) и (1.44) записываем

$$
\left[r_{0}\left(i, x_{0}\right)-i a_{0}\right]+\left[r_{1}\left(i, x_{0}\right)-i a_{1}\right]+\cdots+\left[r_{D}\left(i, x_{0}\right)-i a_{D}\right]=0 .
$$

По аналогии (1.38) с отклонениями $\delta_{1}\left(i, x_{0}\right), \ldots, \delta_{D}\left(i, x_{0}\right)$ определим нулевое цветное отклонение как

$$
\delta_{0}\left(i, x_{0}\right)=r_{0}\left(i, x_{0}\right)-i a_{0} .
$$

Тогда в силу (1.45) и (1.39) для всех цветных отклонений выполняется соотношение

$$
\delta_{0}\left(i, x_{0}\right)+\delta_{1}\left(i, x_{0}\right)+\cdots+\delta_{D}\left(i, x_{0}\right)=0
$$


для всех $i=0,1,2, \ldots$, и, следовательно, для нулевого отклонения получаем представление

$$
\delta_{0}\left(i, x_{0}\right)=-\delta_{1}\left(i, x_{0}\right)-\cdots-\delta_{D}\left(i, x_{0}\right) .
$$

С другой стороны, согласно (1.38) и (1.48) для отклонения $\delta_{0}\left(i, x_{0}\right)$ можем записать

$$
\delta_{0}\left(i, x_{0}\right)=-l_{1}^{*} \cdot \delta\left(i, x_{0}\right)-\cdots-l_{D}^{*} \cdot \delta\left(i, x_{0}\right)=-\left(l_{1}^{*}+\cdots+l_{D}^{*}\right) \cdot \delta\left(i, x_{0}\right) .
$$

Если теперь в дополнение к векторам $l_{1}^{*}, \ldots, l_{D}^{*}$ из (1.36) определить еще вектор $l_{0}^{*}$ равенством

$$
l_{0}^{*}=-l_{1}^{*}-\cdots-l_{D}^{*},
$$

то из (1.49) для нулевого отклонения $\delta_{0}\left(i, x_{0}\right)$ будет следовать представление

$$
\delta_{0}\left(i, x_{0}\right)=l_{0}^{*} \cdot \delta\left(i, x_{0}\right)
$$

Сравнивая его с представлением (1.38), видим, что нам удалось, тем самым, восстановить симметрию между цветными отклонениями относительно всех областей $\mathbb{T}_{0}, \mathbb{T}_{1}, \ldots, \mathbb{T}_{D}$ из разбиения (1.7) тора $\mathbb{T}^{D}$. Причиной нарушения симметрии было первоначальное выделение вектора $v_{0}$ с целью определить базис $l_{1}, \ldots, l_{D}$ решетки $L$ (см. (1.10) и (1.11)).

ПРЕДЛОЖЕНИЕ 1.2. Пусть $L=\mathbb{Z}\left[l_{1}, \ldots, l_{D}\right]$ будет полной решеткой $(1.11), l_{1}^{*}, \ldots, l_{D}^{*}-$ двойственный базис $(1.36) \kappa$ базису $l_{1}, \ldots, l_{D}$ решетки $L$, вектор $l_{0}^{*}$ определен равенством (1.50) $u$

$$
\delta_{k}\left(i, x_{0}\right)=r_{k}\left(i, x_{0}\right)-i a_{k} \quad \text { для } \quad k=0,1, \ldots, D,
$$

где $x_{0}$ - произвольная начальная точка из тора $\mathbb{T}^{D}$, коэфбициенты $a_{k}$ задаются равенствами $a_{k}=-l_{k}^{*} \cdot \alpha$ для $k=1, \ldots, D$ u $a_{0}=1-a_{1}-\cdots-a_{D}$.

Тогда k-отклонения (1.52) удовлетворяют следующему тождеству:

$$
\delta_{0}\left(i, x_{0}\right)+\delta_{1}\left(i, x_{0}\right)+\cdots+\delta_{D}\left(i, x_{0}\right)=0
$$

для всех $i=0,1,2, \ldots$. При этом $k$-отклонения $\delta_{k}\left(i, x_{0}\right)$ и векторное отклонение $\delta\left(i, x_{0}\right)$, определенное равенством (1.19), связаны следующими соотношениями:

$$
\delta_{k}\left(i, x_{0}\right)=l_{k}^{*} \cdot \delta\left(i, x_{0}\right) \quad \partial \Omega \Omega \quad k=0,1, \ldots, D
$$

$u$

$$
\delta\left(i, x_{0}\right)=\delta_{1}\left(i, x_{0}\right) l_{1}+\cdots+\delta_{D}\left(i, x_{0}\right) l_{D}
$$

для всех $i=0,1,2, \ldots$.

Доказательство. Равенства $(1.53)$ и (1.54) для $k$-отклонений $\delta_{k}\left(i, x_{0}\right)$ вытекают из равенств (1.47) и (1.38), (1.51), а разложение (1.55) было доказано в (1.42).

\section{2. Границы для $k$-отклонений}

2.1. Грубые границы для $\boldsymbol{k}$-отклонений. Пусть $x \in \mathbb{R}^{D}$. Определим векторно значную функцию:

$$
\mathcal{D}\left(x, x_{0}\right)=\sum_{0 \leqslant j \leqslant h-1}\left(\operatorname{Fr}\left(j \beta+x_{0}+x\right)-\operatorname{Fr}\left(j \beta+x_{0}\right)\right) .
$$

Из определения (1.15) T-дробной части $\operatorname{Fr}(x)$ вытекает равенство

$$
\mathcal{D}\left(x+l, x_{0}\right)=\mathcal{D}\left(x, x_{0}\right)
$$


для любого вектора $l$ из решетки $L$, т.е. $\mathcal{D}\left(x, x_{0}\right)$ - периодическая функция на пространстве $\mathbb{R}^{D}$ с полной решеткой периодов $L$. В терминах функции $\mathcal{D}\left(x, x_{0}\right)$ равенство $(1.34)$ можем записать как композицию

$$
\delta\left(i, x_{0}\right)=\mathcal{D}\left(\operatorname{Fr}(i \beta), x_{0}\right)
$$

функций $\operatorname{Fr}(x)$ и $\mathcal{D}\left(x, x_{0}\right)$.

Пусть $i>h$. Определим разностную функцию

$$
\Delta(x, y)=\operatorname{Fr}(x+y)-\operatorname{Fr}(y)
$$

обладающую согласно определению (1.15) T-дробной части $\operatorname{Fr}(x)$ свойством периодичности

$$
\Delta\left(x+l, y+l^{\prime}\right)=\Delta(x, y)
$$

с периодами $l, l^{\prime}$ из решетки $L$. Используя $(2.2)$, функцию $\mathcal{D}\left(x, x_{0}\right)$ можно будет переписать в виде

$$
\mathcal{D}\left(x, x_{0}\right)=\sum_{0 \leqslant j \leqslant h-1} \Delta\left(x, j \beta+x_{0}\right)
$$

Из определения (2.2) разностной функции $\Delta(x, y)$ следует, что для любых $x, y \in \mathbb{R}^{D}$ справедливо включение

$$
\Delta(x, y) \in T_{\Delta}
$$

где $T_{\Delta}=\left\{t-t^{\prime}: t, t^{\prime} \in T\right\}-$ разностное множество для развертки $T=T^{D}$. Из определения вытекает, что разностное множество $T_{\Delta}$ центрально-симметрично относительно начала координат в $\mathbb{R}^{D}$. Из (2.3) и (2.4) вытекает включение

$$
\mathcal{D}\left(x, x_{0}\right) \in h T_{\Delta}
$$

для любого $x$ из $\mathbb{R}^{D}$, где умножение на $h$ в правой части $(2.5)$ означает гомотетию $t \mapsto h t$ с коэффициентом $h=1,2,3, \ldots$.

Как следствие из (2.4) для любых $x, y \in \mathbb{R}^{D}$ также получаем последовательность включений

$$
l_{k}^{*} \cdot \Delta(x, y) \in\left(l_{k}^{*} \cdot T\right)_{\Delta} \quad \text { для } \quad k=0,1, \ldots, D,
$$

поскольку $l_{k}^{*} \cdot(T)_{\Delta}=\left(l_{k}^{*} \cdot T\right)_{\Delta}$ в силу равенства $l_{k}^{*} \cdot T=\left\{l_{k}^{*} \cdot t, t \in T\right\}$. Если обозначить

$$
\mathcal{D}_{k}\left(x, x_{0}\right)=l_{k}^{*} \cdot \mathcal{D}\left(x, x_{0}\right),
$$

то из (2.5)-(2.7) для любого $x \in \mathbb{R}^{D}$ получаем включения

$$
\mathcal{D}_{k}\left(x, x_{0}\right) \in h\left(l_{k}^{*} \cdot T\right)_{\Delta} \quad \text { для } \quad k=0,1, \ldots, D .
$$

Для произвольного множества $X \subset \mathbb{R}^{D}$ определим крайние значения:

$$
\underline{m}_{k}(X)=\inf _{x \in X} l_{k}^{*} \cdot x, \quad \bar{m}_{k}(X)=\sup _{x \in X} l_{k}^{*} \cdot x .
$$

Из включений (2.8) и (2.9) следует неравенство

$$
\left|\mathcal{D}_{k}\left(x, x_{0}\right)\right| \leqslant\left(\bar{m}_{k}(T)-\underline{m}_{k}(T)\right) h
$$

для любого $x \in \mathbb{R}^{D}$ и $k=0,1, \ldots, D$. Поскольку согласно $(2.1)$ векторное отклонение $\delta\left(i, x_{0}\right)$ разлагается в композицию $\delta\left(i, x_{0}\right)=\mathcal{D}\left(\operatorname{Fr}(i \beta), x_{0}\right)$, то из $(2.10)$ вытекают неравенства

$$
\left|\delta_{k}\left(i, x_{0}\right)\right| \leqslant c_{k}(T) h \quad \text { для } i>h
$$


с константами

$$
c_{k}(T)=\bar{m}_{k}(T)-\underline{m}_{k}(T),
$$

не зависящими от $h, i$ и определяемыми исключительно размерами развертки тора $T=T^{D}$, определенной в (1.4).

В случае $0 \leqslant i \leqslant h$ рассуждения аналогичны и вместо (2.11) из (1.33) и (2.5) получаем неравнства

$$
\left|\delta_{k}\left(i, x_{0}\right)\right| \leqslant c_{k}(T) i \quad \text { для } \quad 0 \leqslant i \leqslant h .
$$

ТеОРема 2.1. 1) При любом $k=0,1, \ldots, D$ выполняются неравенства

$$
\left|\delta_{k}\left(i, x_{0}\right)\right| \leqslant c_{k}\left(T^{D}\right) h
$$

для всех $i=0,1,2, \ldots$, где $c_{k}\left(T^{D}\right)$ - константы (2.12), не зависящие от $h, i$ и определяемые размерами развертки тора $T^{D}$ из (1.4).

2) Если дополнительно предположить, что $\alpha$ - максимально иррациональный сдвиг (см. $n$. 1.3) и в разбиении (1.4) развертки $T^{D}$ каждая область $T_{k}$ кубируема, то $k$-отклонения $\delta_{k}\left(i, x_{0}\right)$, определенные равенствами (1.52), имеют вид

$$
\delta_{k}\left(i, x_{0}\right)=r_{k}\left(i, x_{0}\right)-i a_{k}
$$

¿de

$$
a_{k}=\frac{\operatorname{vol} T_{k}}{\operatorname{vol} T^{D}}
$$

для $k=0,1, \ldots, D$. Здесъ $\operatorname{vol} T_{k} u \operatorname{vol} T^{D}$ обозначают обгемы областей $T_{k} \subset T^{D}$ и всей развертки тора $T^{D}$.

ДоказАтельство. 1) Неравенства (2.14) следуют из (2.11) и (2.13).

2) Из представления (2.15) и неравенств (2.14) следует

$$
\lim _{i \rightarrow+\infty} \frac{r_{k}\left(i, x_{0}\right)}{i}=a_{k}
$$

В силу (1.20) вектор сдвига $\beta$ также будет максимально иррациональным. Для таких сдвигов тора $S_{\beta}$ известно [12], что

$$
\lim _{i \rightarrow+\infty} \frac{r_{k}\left(i, x_{0}\right)}{i}=\frac{\operatorname{vol} T_{k}}{\operatorname{vol} T^{D}} .
$$

Отсюда и из (2.17) вытекает равенство (2.16).

2.2. Вырождение орбит. Пусть вектор сдвига $\alpha$ будет произвольным, т.е. не обязательно иррациональным, и пусть $\operatorname{Orb}_{S_{\beta}}\left(x_{0}\right)$ - орбита (1.41) произвольной начальной точки $x_{0} \in T$ относительно сдвига $S_{\beta}$, где вектор сдвига $\beta$ определен равенством (1.20). Обозначим через $\overline{\mathrm{Orb}}_{S_{\beta}}\left(x_{0}\right)$ замыкание орбиты $\operatorname{Orb}_{S_{\beta}}\left(x_{0}\right)$.

Если вектор сдвига $\alpha$ будет максимально иррациональным, т.е. $\operatorname{rank}_{\mathbb{Z}}(\alpha)=D$, то вектор $\beta$, как мы уже отмечали, также будет максимально иррациональным. Из теоремы Вейля [12] следует, что для указанных $\beta$ будет выполняться равенство

$$
\operatorname{dim} \overline{\mathrm{Orb}}_{S_{\beta}}\left(x_{0}\right)=D
$$

и, таким образом, в этом случае выполняется формула

$$
\overline{\mathrm{Orb}}_{S_{\beta}}\left(x_{0}\right)=\bar{T} .
$$

В общем случае равенство (2.19) нужно заменить на включение

$$
\overline{\mathrm{Orb}}_{S_{\beta}}\left(x_{0}\right) \subseteq \bar{T},
$$


поэтому для констант (2.12) из (2.20) будет следовать неравенство

$$
c_{k}(X) \leqslant c_{k}(T)
$$

Здесь множество $X$ определяется с помощью биекции (1.3):

$$
T^{D} \supseteq X \longrightarrow \overline{\operatorname{Orb}}_{S_{\beta}}\left(x_{0}\right) \subset \mathbb{T}^{D}
$$

Если неравенство (2.21) будет строгим, то как следствие в случае произвольного вектора сдвига $\alpha$ мы получим для отклонений $\delta_{k}\left(i, x_{0}\right)$ усиление неравенства $(2.14)$.

Повторяя доказательство теоремы 2.1, получаем следующее утверждение.

ТЕОРема 2.2. При любом $k=0,1, \ldots, D$ выполняются неравенства

$$
\left|\delta_{k}\left(i, x_{0}\right)\right| \leqslant c_{k}(X) h
$$

для всех $i=0,1,2, \ldots$, где константы $c_{k}(X)$ вычисляются по формуле

$$
c_{k}(X)=\bar{m}_{k}(X)-\underline{m}_{k}(X),
$$

не зависят от $i$ и определяются исключительно размерами множества $X$ из (2.22). Константы $\bar{m}_{k}(X)$ и $\underline{m}_{k}(X)$ определены в (2.9).

2.3. Точные границы для $\boldsymbol{k}$-отклонений. В начале докажем общую формулу для $k$-отклонений.

ПРЕДЛОЖЕНИЕ 2.1. 1) Пусть вектор сдвига $\beta$ определен равенством (1.20) и k-отклонения

$$
\delta_{k}\left(i, x_{0}\right)=r_{k}\left(i, x_{0}\right)-i a_{k} \quad \text { для } \quad k=0,1, \ldots, D
$$

определены в (1.38) и (1.51). Тогда k-отклонения могут быть представлены еще в одном из двух видов:

$$
\delta_{k}\left(i, x_{0}\right)=\sum_{0 \leqslant j<i}\left(\operatorname{Fr}_{k}\left(j \beta+h \beta+x_{0}\right)-\operatorname{Fr}_{k}\left(j \beta+x_{0}\right)\right)
$$

$\partial л я 0 \leqslant i \leqslant h u$

$$
\delta_{k}\left(i, x_{0}\right)=\sum_{0 \leqslant j \leqslant h-1}\left(\operatorname{Fr}_{k}\left(j \beta+i \beta+x_{0}\right)-\operatorname{Fr}_{k}\left(j \beta+x_{0}\right)\right)
$$

для $i>h$. Здесъ

$$
\operatorname{Fr}_{k}(x)=l_{k}^{*} \cdot \operatorname{Fr}(x)
$$

для $k=0,1, \ldots, D$ u $\operatorname{Fr}(x)=\operatorname{Fr}_{T}(x)$ обозначает векторную $T$-дробную часть (2.1) точки $x \in \mathbb{R}^{D}$.

2) В случае $h=1$, когда вектор сдвига $\beta=\alpha$, формуль (2.25) и (2.26) принимают более простой вид

$$
\delta_{k}\left(i, x_{0}\right)=\operatorname{Fr}_{k}\left(i \alpha+x_{0}\right)-\operatorname{Fr}_{k}\left(x_{0}\right)
$$

для $k=0,1, \ldots, D$ и любого $i=0,1,2, \ldots$.

ДокАЗАтельство. Равенство (2.25) вытекает непосредственно из определения (1.19) и формул для $k$-отклонений (1.37), (1.51), а равенство $(2.26)$ - из (1.34). 
2.4. Единичный базис. По условию (1.12) векторы $l_{1}, \ldots, l_{D}$ линейно независимы над $\mathbb{R}$, поэтому существует линейное отображение $A$ такое, что $A l_{k}=-\mathbf{e}_{k}$ для $k=1,2, \ldots, D$, где

$$
\mathbf{e}_{1}=(1,0, \ldots, 0), \quad \ldots, \quad \mathbf{e}_{D}=(0,0, \ldots, 1)
$$

- единичный базис. Так как преобразование $A$ невырожденное, то при переходе от развертки $T$ к $A T$ сохраняются все приведенные выше рассуждения из данного раздела. Поэтому, не уменьшаяя общности, можем считать, что

$$
l_{1}=-\mathbf{e}_{1}, \quad \ldots, \quad l_{D}=-\mathbf{e}_{D} .
$$

Тогда решетка $L$, определенная в (1.11), будет иметь вид

$$
L=\mathbb{Z}^{D}=\mathbb{Z}\left[\mathbf{e}_{1}, \ldots, \mathbf{e}_{D}\right],
$$

т.е. будет кубической решеткой $\mathbb{Z}^{D}$ в пространстве $\mathbb{R}^{D}$.

ТЕОРемА 2.3. Пусть векторы $l_{1}, \ldots, l_{D}$ выбраны в виде (2.29) и $x_{0}$ - произволъная начальная точки из тора $\mathbb{T}^{D}$.

1) Тогда k-отклонения (1.39) и (1.46) принимают вид

$$
\delta_{k}\left(i, x_{0}\right)=r_{k}\left(i, x_{0}\right)-i \alpha_{k} \quad \text { для } \quad k=0,1, \ldots, D,
$$

где вектор сдвига $\alpha=\left(\alpha_{1}, \ldots, \alpha_{D}\right)$ записан в единичном базисе $\mathbf{e}_{1}, \ldots, \mathbf{e}_{D} u$

$$
\alpha_{0}=1-\alpha_{1}-\cdots-\alpha_{D}
$$

2) Пусть выбран вектор сдвига $\beta=(\alpha+l) / h$ с произвольным натуральным $h$ и вектором $l$ из кубической решетки $\mathbb{Z}^{D}$. Тогда при любом $k=0,1, \ldots, D$ выполняются неравенства

$$
\left|\delta_{k}\left(i, x_{0}\right)\right| \leqslant c_{k}(X) h
$$

для всех $i=0,1,2, \ldots$ с константами $c_{k}(X)=\bar{m}_{k}(X)-\underline{m}_{k}(X)$, где множество $X$ определено в $(2.22)$,

$$
\underline{m}_{k}(X)=-\sup _{x \in X} \mathbf{e}_{k} \cdot x, \quad \bar{m}_{k}(X)=-\inf _{x \in X} \mathbf{e}_{k} \cdot x
$$

$\partial л я k=1, \ldots, D u$

$$
\underline{m}_{0}(X)=\inf _{x \in X} \mathbf{e}_{0} \cdot x, \quad \bar{m}_{0}(X)=\sup _{x \in X} \mathbf{e}_{0} \cdot x
$$

для $k=0$, где $\mathbf{e}_{0}=\mathbf{e}_{1}+\cdots+\mathbf{e}_{D}$.

3) В случае $h=1$ выполняются точные неравенства

$$
x_{0 k}-\sup _{x \in X} \mathbf{e}_{k} \cdot x \leqslant \delta_{k}\left(i, x_{0}\right) \leqslant x_{0 k}-\inf _{x \in X} \mathbf{e}_{k} \cdot x
$$

для $k=1, \ldots, D$, где начальная точка $x_{0}=\left(x_{01}, \ldots, x_{0 D}\right)$ записана в базисе $\mathbf{e}_{1}, \ldots, \mathbf{e}_{D}, u$

$$
\underline{m}_{0}(X)-\sigma\left(x_{0}\right) \leqslant \delta_{0}\left(i, x_{0}\right) \leqslant \bar{m}_{0}(X)-\sigma\left(x_{0}\right),
$$

где $\sigma\left(x_{0}\right)=x_{01}+\cdots+x_{0 D}$.

ДокАЗАТЕЛЬСтво. 1) Согласно (1.40) и (2.29) имеем $a_{k}=-l_{k}^{*} \cdot \alpha=\mathbf{e}_{k} \cdot \alpha=\alpha_{k}$ для $k=1, \ldots, D$. Отсюда и из (1.44) для отклонений $\delta_{k}\left(i, x_{0}\right)$ вытекает формула $(2.31)$.

2) Неравенство (2.32) следует из теоремы 2.2 и формулы (2.9) для крайних значений $\underline{m}_{k}(X)$ и $\bar{m}_{k}(X)$. 
3) Пусть $h=1$. По формуле (2.28) имеем

$$
\delta_{k}\left(i, x_{0}\right)=\operatorname{Fr}_{k}\left(i \alpha+x_{0}\right)-\operatorname{Fr}_{k}\left(x_{0}\right)
$$

для $k=0,1, \ldots, D$. В этом представлении согласно формуле $(2.27)$

$$
\operatorname{Fr}_{k}(x)=l_{k}^{*} \cdot \operatorname{Fr}(x)=-\mathbf{e}_{k} \cdot \operatorname{Fr}(x)
$$

для $k=1, \ldots, D$ и

$$
\operatorname{Fr}_{0}(x)=l_{0}^{*} \cdot \operatorname{Fr}(x)=\mathbf{e}_{0} \cdot \operatorname{Fr}(x),
$$

так как $l_{k}^{*}=-\mathbf{e}_{k}$ для $k=1, \ldots, D$, и в силу равенства (1.50) имеем

$$
l_{0}^{*}=-l_{1}^{*}-\cdots-l_{D}^{*}=\mathbf{e}_{1}+\cdots+\mathbf{e}_{D}=\mathbf{e}_{0} .
$$

По определению (1.15) векторной дробной части $\operatorname{Fr}(x)$ можем записать, что

$$
\operatorname{Fr}(x)=x \quad \text { для } \quad x \in T^{D},
$$

где $T^{D}$ - развертка тора (1.4). Пусть вектор $x=\left(x_{1}, \ldots, x_{D}\right)$ записан в единичном базисе $\mathbf{e}_{1}, \ldots, \mathbf{e}_{D}$. Тогда из $(2.36),(2.37)$ и $(2.39)$ получаем равенства

$$
\operatorname{Fr}_{k}(x)=-x_{k}
$$

для $k=1, \ldots, D$ и еще одно равенство

$$
\operatorname{Fr}_{0}(x)=x_{1}+\cdots+x_{D}=\sigma(x)
$$

в случае $k=0$. Пусть сначала $k=1, \ldots, D$. Тогда по формулам $(2.35)$ и $(2.40)$ для отклонений $\delta_{k}\left(i, x_{0}\right)$ получаем представление

$$
\delta_{k}\left(i, x_{0}\right)=x_{0 k}-x_{k},
$$

где $x_{0}=\left(x_{01}, \ldots, x_{0 D}\right)$ и точки $x=i \alpha+x_{0}=\left(x_{1}, \ldots, x_{D}\right)$ принадлежат множеству $X \subseteq T^{c}$. Следовательно, для них выполняются неравенства

$$
\inf _{x \in X} \mathbf{e}_{k} \cdot x \leqslant x_{k} \leqslant \sup _{x \in X} \mathbf{e}_{k} \cdot x
$$

Из (2.42) и (2.43) вытекают неравенства (2.33). Они являются точными, так как указанные точки $x$ при $i \rightarrow+\infty$ всюду плотно заполняет множество $X$.

В оставшемся случае $k=0$ в силу $(2.35)$ и $(2.41)$ для отклонения $\delta_{0}\left(i, x_{0}\right)$ получаем другое представление

$$
\delta_{0}\left(i, x_{0}\right)=\mathbf{e}_{0} \cdot x-\sigma(x),
$$

из которого вытекают неравенства (2.34).

\section{3. Разбиения вытянутых параллелоэдров}

3.1. Вытянутые кубы $\boldsymbol{C}_{\boldsymbol{s}}$. Приведем основные понятия и результаты из [9], необходимые нам для дальнейшего. Пусть $C=C^{D}$ - замкнутый $D$-мерный куб, натянутый на единичные векторы $\mathbf{e}_{1}, \ldots, \mathbf{e}_{D}$. Обозначим через $\mathbb{R}_{+}^{D}$ положительный конус, состоящий из $x=\left(x_{1}, \ldots, x_{D}\right) \in \mathbb{R}^{D}$ с координатами $x_{1}>0, \ldots, x_{D}>0$. Для любого вектора $\mathbf{s}$ из конуса $\mathbb{R}_{+}^{D}$ определим операцию вытягивания $\operatorname{Str}_{\mathbf{s}}$ произвольного множества $X \subset \mathbb{R}^{D}$, полагая

$$
\operatorname{Str}_{\mathbf{s}}(X)=\bigcup_{t \in I}(X+t \mathbf{s})
$$


где $I=[0,1]$, при этом $\mathbf{s}$ будем называть вытягивющим вектором. Операцию вытягивания (3.1) также можно записать через сумму по Минковскому:

$$
\operatorname{Str}_{\mathbf{s}}(X)=X+\mathbf{s} \cdot I
$$

Применяя данную операцию к единичному кубу $C$, построим из него многогранник

$$
C_{\mathbf{s}}=\operatorname{Str}_{\mathbf{s}}(C),
$$

представляющий собой вытянутый куб, имеющий объем vol $C_{\mathbf{s}}=\sigma(\mathbf{s})+1$. Здесь мы обозначили $\sigma(\mathbf{s})=s_{1}+\cdots+s_{D}$ для вектора $\mathbf{s}=\left(s_{1}, \ldots, s_{D}\right)$ (см. [8]).

Далее мы будем использовать понятие разбиения множеств в обычном смысле, когда рассматриваются покрытия множеств $X=X_{1} \sqcup X_{2} \sqcup \cdots$ с условием $X_{k} \cap X_{k^{\prime}}=\varnothing$ для $k \neq k^{\prime}$, а также в расширенном смысле $X=X_{1} \cup X_{2} \cup \cdots$, предполагая, что различные множества $X_{k}$ не имеют общих внутренних точек.

Вытянутые кубы $C_{\mathbf{s}}$ обладают следующим важным для наших целей свойством [9, теорема 1.1]: для любого вектора $\mathbf{s} \in \mathbb{R}_{+}^{D}$ имеет место разбиение пространства

$$
\mathbb{R}^{D}=\bigcup_{l \in L_{\mathbf{s}}} C_{\mathbf{s}}[l],
$$

где $C_{\mathbf{s}}[l]=C_{\mathbf{s}}+l-$ множество, полученное сдвигом куба $C_{s}$ на вектор $l, u L_{\mathbf{s}}=$ $\mathbb{Z}\left[\mathbf{e}_{1, s}, \ldots, \mathbf{e}_{D, s}\right]-$ полная решетка, порождаемая векторами $\mathbf{e}_{k, \mathbf{s}}=\mathbf{e}_{k}+\mathbf{s} \partial л я k=1, \ldots, D$.

Таким образом, вытянутые кубы $C_{\mathbf{s}}$ трансляционно разбивают пространство $\mathbb{R}^{D}$ с помощью трансляционной решетки $L_{\mathbf{s}}$.

3.2. Вытянутые параллелоэдры $\mathcal{C}_{\mathbf{c}}$. Линейное отображение

$$
\mathbb{R}^{D \stackrel{M_{\mathbf{s}}}{\longrightarrow}} \mathbb{R}^{D}: \quad \mathbf{e}_{k, \mathbf{s}} \mapsto \mathbf{e}_{k}, \quad k=1, \ldots, D
$$

задает изоморфизм решеток $M_{\mathbf{s}}: L_{\mathbf{s}}^{+} \rightarrow \mathbb{Z}^{D}$ и переводит $D$-мерный единичный куб $C$ в параллелоэдр - параллелепипед

$$
\mathcal{C}=M_{\mathbf{s}}(C),
$$

натянутый соответственно на векторы $\mathbf{e}_{1, \mathbf{c}}=\mathbf{e}_{1}-\mathbf{c}, \ldots, \mathbf{e}_{D, \mathbf{c}}=\mathbf{e}_{D}-\mathbf{c}$, где вектор $\mathbf{c}$ вычисляется по формуле

$$
\mathbf{c}=M_{\mathbf{s}}(\mathbf{s})
$$

поскольку согласно (3.5) $M_{\mathbf{s}}\left(\mathbf{e}_{k}\right)=M_{\mathbf{s}}\left(\mathbf{e}_{k, \mathbf{s}}-\mathbf{s}\right)=\mathbf{e}_{k}-\mathbf{c}$. Пусть $\mathbf{s}=s_{1} \mathbf{e}_{1}+\cdots+s_{D} \mathbf{e}_{D}$. Тогда из последнего равенства получаем $s_{1} M_{s}\left(\mathbf{e}_{1}\right)+\cdots+s_{D} M_{s}\left(\mathbf{e}_{D}\right)=\mathbf{s}-\sigma(\mathbf{s}) \mathbf{c}$. Так как левая часть последнего равенства равна $M_{\mathbf{s}}(\mathbf{s})=\mathbf{c}$, то записываем $\mathbf{c}=\mathbf{s}-\sigma(\mathbf{s}) \mathbf{c}$. Отсюда для вектора $\mathbf{c}$ из (3.7) выводим явную формулу

$$
\mathbf{c}=\frac{\mathbf{s}}{\sigma(\mathbf{s})+1} .
$$

Согласно (3.6) вытянутый куб $C_{\mathbf{s}}$ отображается $M_{\mathbf{s}}: C_{\mathbf{s}} \rightarrow \mathcal{C}_{\mathbf{c}}$ в многогранник

$$
\mathcal{C}_{\mathbf{c}}=\operatorname{Str}_{\mathbf{c}}(\mathcal{C})
$$

получающийся вытягиванием параллелоэдра $\mathcal{C}$ вдоль вектора $\mathbf{c}$, связанного с вектором $\mathbf{s}$ формулой (3.8). Вытянутый параллелоэдр $\mathcal{C}_{\mathbf{c}}$ имеет объем

$$
\operatorname{vol} \mathcal{C}_{\mathbf{c}}=1
$$

и число вершин $\# V\left(\mathcal{C}_{\mathbf{c}}\right)=2^{D+1}-2($ см. [8]). 
3.3. Вытягивающие векторы для параллелоэдров. Согласно формуле (3.8) отображения, переводящие вытягивающие векторы $\mathbf{s}$ в векторы $\mathbf{c}$, имеют вид

$$
\operatorname{inv}(x)=\frac{x}{\sigma(x)+1}
$$

и представляют собою линейно-инверсные преобразования пространства $\mathbb{R}^{D}$. Для наших целей мы ограничим инверсию inv на положительный конус $\mathbb{R}_{+}^{D} \subset \mathbb{R}^{D}$.

Имеет место следующая биекция [10, лемма 3.1]:

$$
\text { inv: } \mathbb{R}_{+}^{D} \longrightarrow \mathbf{C}_{<1} \text {. }
$$

Здесь

$$
\mathbf{C}_{<1}=\left\{\mathbf{c} \in \mathbb{R}_{+}^{D}: \sigma(\mathbf{c})<1\right\}
$$

где $\sigma(\mathbf{c})=c_{1}+\cdots+c_{D}$. Замыкание множества $\mathbf{C}_{<1}$ является $D$-мерным симплексом, который содержит $(D-1)$-мерный симплекс $\overline{\mathbf{C}}_{1}=\left\{\mathbf{c} \in \overline{\mathbb{R}}_{+}^{D}: \sigma(\mathbf{c})=1\right\}$.

В [10] доказана следующая теорема.

Tеорема 3.1. 1) Множество

$$
\mathbf{C}_{\leqslant 1}=\mathbf{C}_{<1} \sqcup \mathbf{C}_{1},
$$

где $\mathbf{C}_{1}=\left\{\mathbf{c} \in \mathbb{R}_{+}^{D}: \sigma(\mathbf{c})=1\right\}$ - внутренность симплекса $(1.38)$, представляет собой множество вытягивающих векторов для параллелоэдров (3.9): любому вектору $\mathbf{c} \in \mathbf{C}_{\leqslant 1}$ cоответствует $\mathbf{c} \mapsto \mathcal{C}_{\mathbf{c}}-$ выпуклый параллелоэдр $\mathcal{C}_{\mathbf{c}}$.

2) Вытянутые параллелоэдры $\mathcal{C}_{\mathbf{c}}$ разбивают пространство

$$
\mathbb{R}^{D}=\bigcup_{l \in \mathbb{Z}^{D}} \mathcal{C}_{\mathbf{c}}[l]
$$

на многогранники $\mathcal{C}_{\mathbf{c}}[l]=\mathcal{C}_{\mathbf{c}}+l$ без общих внутренних точек.

3.4. Разбиения и перекладывания вытянутых параллелоэдров. Используя (3.14), определим разбиение пространства $\mathbb{R}^{D}$ :

$$
\mathcal{T}_{\mathbf{c}}=\bigcup_{l \in \mathbb{Z}^{D}} \mathcal{C}_{\mathbf{c}}[l]
$$

Обозначим через

$$
\partial \mathcal{T}_{\mathbf{c}}=\bigcup_{l \in \mathbb{Z}^{D}} \partial \mathcal{C}_{\mathbf{c}}[l]
$$

множество всех границ разбиения $\mathcal{T}_{\mathbf{c}}$, состоящее из границ всех многогранников $\mathcal{C}_{\mathbf{c}}[l]$. Для любого $\alpha=\lambda \mathbf{c}, 0<\lambda<1$, определим следующее множество границ:

$$
\partial \mathcal{C}_{\mathbf{c}, \lambda}=\partial \mathcal{C}_{\mathbf{c}} \cup\left[\mathcal{C}_{\mathbf{c}} \cap\left(\partial \mathcal{T}_{\mathbf{c}}-\alpha\right)\right] \subset \mathcal{C}_{\mathbf{c}}
$$

Границы $\partial \mathcal{C}_{\mathbf{c}, \lambda}$ разбивают вытянутый параллелоэдр $\mathcal{C}_{\mathbf{c}}$ на $D+1$ областей без общих внутренних точек [10]:

$$
\mathcal{C}_{\mathbf{c}, \lambda}=\overline{\mathcal{P}}_{0} \cup \cdots \cup \overline{\mathcal{P}}_{D}
$$

где $\overline{\mathcal{P}}_{k}$ для $k=1, \ldots, D$ - параллелоэдры, содедержащие точки $e_{k}=(0, \ldots, \stackrel{(k)}{1}, \ldots, 0)$, a

$$
\overline{\mathcal{P}}_{0}=\overline{\left[\mathcal{C}_{c} \backslash\left(\overline{\mathcal{P}}_{1} \cup \cdots \cup \overline{\mathcal{P}}_{D}\right)\right]}
$$


- вытянутый параллелоэдр вида $\overline{\mathcal{P}}_{0}=\operatorname{Str}_{\mathbf{c}-\alpha}(\mathcal{C})$. Отметим, что $\mathcal{C}_{\mathbf{c}, \lambda}$ - это тот же самый параллелоэдр $\mathcal{C}_{\mathbf{c}}$, определенный в $(3.9)$, но уже разбитый $(3.17)$ на $D+1$ многограннков $\overline{\mathcal{P}}_{0}, \ldots, \overline{\mathcal{P}}_{D}$. Здесь мы через $\overline{\mathcal{P}}_{k}$ обозначаем замкнутые многогранники. Для дальнейшего же нам потребуются не сами $\overline{\mathcal{P}}_{k}$, а некоторые незамкнутые многогранники $\mathcal{P}_{k} \subset \overline{\mathcal{P}}_{k}$, которые будут определены в п. 4.2.

Поясним приведенную выше конструкцию на примере небольших размерностей $D$. Так, в случае $D=1$ множество границ $\partial \mathcal{C}_{\mathbf{c}, \lambda}(3.16)$ состоит из трех точек, две из которых являются концами интервала $\mathcal{C}_{\mathbf{c}}$, а оставшаяся делит его на две части: $\mathcal{C}_{\mathbf{c}, \lambda}=\overline{\mathcal{P}}_{0} \cup \overline{\mathcal{P}}_{1}$. Если $D=2$, то границы $\partial \mathcal{C}_{\mathbf{c}, \lambda}$ разбивают шестиугольник $\mathcal{C}_{\mathbf{c}}$ на меньший шестиугольник и два параллелограмма с общей стороной: $\mathcal{C}_{\mathbf{c}, \lambda}=\overline{\mathcal{P}}_{0} \cup \overline{\mathcal{P}}_{1} \cup \overline{\mathcal{P}}_{2}$. Если же $D=3$, то $\mathcal{C}_{\mathbf{c}, \lambda}=\overline{\mathcal{P}}_{0} \cup \overline{\mathcal{P}}_{1} \cup \overline{\mathcal{P}}_{2} \cup \overline{\mathcal{P}}_{3}$ представляет собою разбиение ромбического додекаэдра $\mathcal{C}_{\mathbf{c}}$ на такой же ме́ньший додекаэдр и три параллелепитеда, попарно имеющих по одной общей грани и общее для всех ребро. Случай произвольной размерности $D$ разобран в [9], [10].

Зададим перекладывание

$$
S_{v}: \mathcal{C}_{\mathbf{c}, \lambda} \longrightarrow \mathcal{C}_{\mathbf{c}, \lambda}
$$

вытянутого параллелоэдра $\mathcal{C}_{\mathbf{c}, \lambda}$, имеющего разбиение $(3.17)$, с помощью параллельных сдвигов

$$
S_{v}\left(\overline{\mathcal{P}}_{k}\right)=\overline{\mathcal{P}}_{k}\left[v_{k}\right]=\overline{\mathcal{P}}_{k}+v_{k}
$$

многогранников $\overline{\mathcal{P}}_{k}$ из разбиения (3.17) соответственно на векторы

$$
v_{k}=\alpha- \begin{cases}0, & \text { если } k=0, \\ \mathbf{e}_{k}, & \text { если } k>0 .\end{cases}
$$

Перекладывание $S_{v}$ индуцирует многозначное отображение

$$
S_{v}: \operatorname{Str}_{\mathbf{c}}(\mathcal{C}) \longrightarrow \mathbb{R}^{D}
$$

определяемое условиями $x \mapsto S_{v}(x)=x+v_{k}$, если $x \in \overline{\mathcal{P}}_{k}$.

Из [9, предложение 1.1] вытекает следующее свойство перекладывания $S_{v}$.

ПРЕДЛОЖЕНИЕ 3.1. Вытянутый параллелоэдр $\mathcal{C}_{\mathbf{c}, \lambda}=\operatorname{Str}_{\mathbf{c}}(\mathcal{C})$ с разбиением (3.17) замкнут $S_{v}\left(\mathcal{C}_{\mathbf{c}, \lambda}\right) \subseteq \mathcal{C}_{\mathbf{c}, \lambda}$ относительно операчии перекладывания (3.18).

Из определения (3.19) векторов сдвигов $v_{k}$ вытекают сравнения $v_{k} \equiv \alpha \bmod \mathbb{Z}^{D}$ для любого $k=0,1, \ldots, D$. Отсюда и из (3.20) получаем сравнение

$$
S_{v}(x) \equiv x+\alpha \bmod \mathbb{Z}^{D},
$$

означающее, что многозначное отображение $S_{v}$ становится однозначным на вытянутом параллелоэдре $S_{v}: \mathcal{C}_{\mathbf{c}} \rightarrow \mathcal{C}_{\mathbf{c}}$, если отображение $S_{v}$ рассмотреть по $\bmod \mathbb{Z}^{D}$.

Определим на торе $\mathbb{T}^{D} \simeq \mathbb{R}^{D} / \mathbb{Z}^{D}$ сдвиг

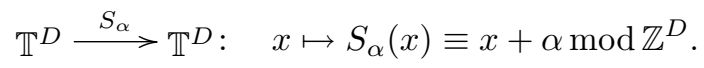

Тогда из (3.18)-(3.21) вытекает, что коммутативна следующая диаграмма:

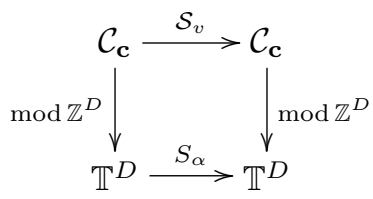




\section{4. Перекладывающиеся торические развертки: продолжение}

4.1. Определение перекладывающихся торических разверток. Назовем подмножество $T^{D} \subset \mathbb{R}^{D}$ перекладывающейся торической разверткой, если оно удовлетворяет следующим условиям:

1) множество $T^{D}$ ограниченно;

2) задано разбиение

$$
T^{D}=T_{0} \sqcup T_{1} \sqcup \cdots \sqcup T_{D}
$$

на непересекающиеся множества $T_{k}$;

3) с помощью разбиения (4.1) и некоторой фиксированной системы $v=\left\{v_{0}, v_{1}, \ldots, v_{D}\right\}$ векторов из $\mathbb{R}^{D}$ задано перекладывание $\mathcal{S}_{v}\left(T^{D}\right) \stackrel{\text { def }}{=} \mathcal{S}_{v}\left(T_{0}\right) \cup \mathcal{S}_{v}\left(T_{1}\right) \cup \cdots \cup \mathcal{S}_{v}\left(T_{D}\right)$, где $\mathcal{S}_{v}\left(T_{k}\right)=T_{k}\left[v_{k}\right]$

4) множество $T^{D}$ замкнуто относительно перекладывания $\mathcal{S}_{v}$, т.е. выполняется включение $\mathcal{S}_{v}\left(T^{D}\right) \subseteq T^{D}$

5) отображение факторзации

$$
T^{D \stackrel{\bmod L_{v}}{\longrightarrow}} \mathbb{T}_{v}^{D}: \quad x \mapsto x \bmod L_{v}
$$

задает биекцию между $T^{D}$ и тором $\mathbb{T}_{v}^{D} \simeq \mathbb{R}^{D} / L_{v}$, где $L_{v}=\mathbb{Z}\left[l_{1}, \ldots, l_{D}\right]$ - полная решетка из $\mathbb{R}^{D}$ с базисом $l_{k}=v_{k}-v_{0}$ для $k=1, \ldots, D$;

6) коммутативна диаграмма

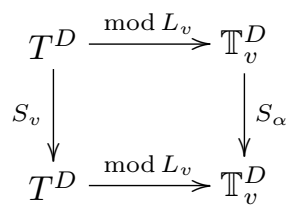

где $S_{v}$ - отображение $S_{v}(x)=x+v_{k}$, если $x \in T_{k}$, индуцированное перекладыванием $\mathcal{S}_{v}$, и $S_{\alpha}(x)=x+\alpha \bmod L_{v}-$ сдвиг тора $\mathbb{T}_{v}^{D}$ на вектор $\alpha \equiv v_{0} \bmod L_{v}$.

4.2. Построение перекладывающихся торических разверток. Чтобы построить для вытянутого параллелотопа $\mathcal{C}_{\mathbf{c}, \lambda}$ с разбиением (3.17) соответствующую ему перекладывающуюся торическую развертку $T_{\mathbf{c}, \lambda} \subset \mathbb{R}^{D}$, нам потребуется следующий алгоритм [9], определяющий индекс $i(x)$ точек $x \in \mathcal{C}_{\mathbf{c}, \lambda}$ в случае максимально иррационального сдвига $\alpha=\left(\alpha_{1}, \ldots, \alpha_{D}\right)$ (см. определение п. 1.3).

$i$-Алгоритм. Шаг 1. Для точки $x \in \mathcal{C}_{\mathbf{c}, \lambda}^{\text {int }}$ полагаем

$$
i(x)=k, \quad \text { где } k-\text { наименьший номер с условием } x \in \mathcal{P}_{k}^{c} \text {. }
$$

Тем самым, мы определили индексы $i(x)$ для всех внутренних точек $x$ из $\mathcal{C}_{\mathbf{c}, \lambda}$.

Шаг 2. Пусть точка $x$ принадлежит границе $\partial \mathcal{C}_{\mathbf{c}, \lambda}$ многогранника $\mathcal{C}_{\mathbf{c}, \lambda}$, и пусть существует точка $x^{\prime} \in \mathcal{C}_{\mathbf{c}, \lambda}^{\text {int }}$ с условием

$$
S_{v}\left(x^{\prime}\right)=x .
$$

Тогда индекс $i(x)$ также определяем по правилу (4.3).

Шаг 3 . Пусть $x_{1}=x$ - та же самая точка, что была на шаге 2 . Так как индекс точки $x_{1}$ уже определен, то однозначно определен ее образ $x_{2}=S_{v}\left(x_{1}\right)$. Если $x_{2} \in \partial \mathcal{C}_{\mathbf{c}, \lambda}$, то приписываем ей индекс $i\left(x_{2}\right)$ по правилу (4.3). Затем рассматриваем точку $x_{3}=S_{v}\left(x_{2}\right)$ и т.д. Если на каком-то шаге $x_{j}=S_{v}\left(x_{j-1}\right)$ окажется внутренней точкой $\mathcal{C}_{\mathbf{c}, \lambda}$, то для нее индекс $i\left(x_{j}\right)$ уже был определен на шаге 1. 
Шаг 4. Пусть точка $y \in \partial \mathcal{C}_{\mathbf{c}, \lambda}$ не удовлетворяет условию (4.4) и не попадает ни в какую $S_{v}$-орбиту точек $x \in \partial \mathcal{C}_{\mathbf{c}, \lambda}$ с условием (4.4). Таким точкам припишем индекс $i(y)=-1$.

Из $i$-алгоритма вытекает, что любая точка $x$ из вытянутого параллелоэдра $\mathcal{C}_{\mathbf{c}, \lambda}$ имеет однозначно определенный индекс $i(x)=-1,0,1, \ldots, D$. Используя индекс $i(x)$, определим следующие множества:

$$
\mathcal{P}_{k}=\left\{x \in \mathcal{C}_{\mathbf{c}, \lambda}: i(x)=k\right\}
$$

для $k=0,1, \ldots, D$. Из однозначности индекса $i(x)$ следует $\mathcal{P}_{k_{1}} \cap \mathcal{P}_{k_{2}}=\varnothing$ с номерами $k_{1} \neq k_{2}$.

Tеорема 4.1. Пусть $\mathbf{c} \in \mathbf{C}_{\leqslant 1}$ u вектор сдвига выбран в виде $\alpha=\lambda \mathbf{c}$, где $0<\lambda \leqslant 1$ в случае $\mathbf{c} \in \mathbf{C}_{<1}$ и $0<\lambda<1$, если $\mathbf{c} \in \mathbf{C}_{1}$. Кроме того, пусть $\mathcal{P}_{k}-$ многогранники (4.5). Тогда их объединение

$$
T_{\mathbf{c}, \lambda}^{D}=\mathcal{P}_{0} \sqcup \mathcal{P}_{1} \sqcup \cdots \sqcup \mathcal{P}_{D}
$$

является перекладывающейся торической разверткой из $\mathbb{R}^{D}$.

ДокАзАтЕльство. Для максимально иррационального вектора сдвига $\operatorname{rank}_{\mathbb{Z}}(\alpha)=D$ теорема была доказана [9, теорема 1.2].

Если же $\operatorname{rank}_{\mathbb{Z}^{D}}(\alpha)<D$, то $\varepsilon$-шевелением $\alpha \rightarrow \alpha_{\varepsilon}:\left\|\alpha-\alpha_{\varepsilon}\right\|<\varepsilon$ можно добиться, чтобы выполнялось условие $\operatorname{rank}_{\mathbb{Z} D}(\alpha)=D$. Далее, по построению (3.17) разбиения $\mathcal{C}_{\mathbf{c}, \lambda}$, а значит, и разбиения $T_{\mathbf{c}, \lambda}^{D}$ непрерывно зависимы от вектора сдвига $\alpha$. Отсюда следует, что утверждение теоремы выполняется для всех векторов сдвига $\alpha \in \mathbb{T}^{d}, \alpha \neq 0$.

Итак, мы доказали, что любому вытянутому параллелоэдру $\mathcal{C}_{\mathbf{c}}$ с разбиением $\mathcal{C}_{\mathbf{c}, \lambda}$ из $(3.17)$ $i$-алгоритм ставит в соответствие перекладывающуюся торическую развертку $T_{\mathbf{c}, \lambda}^{D} \subset \mathcal{C}_{\mathbf{c}} \mathrm{c}$ разбиением (4.6):

$$
\mathcal{C}_{\mathbf{c}} \Longrightarrow \mathcal{C}_{\mathbf{c}, \lambda} \Longrightarrow T\left(\mathcal{C}_{\mathbf{c}, \lambda}\right)=T_{\mathbf{c}, \lambda}^{D}
$$

Ее роль состоит в том, что посредством биекции (4.2) она задает каноническое разбиение тора

$$
\mathbb{T}_{\mathbf{c}, \lambda}^{D}=\mathbb{T}_{0}^{D} \sqcup \mathbb{T}_{1}^{D} \sqcup \cdots \sqcup \mathbb{T}_{D}^{D}
$$

на множества $\mathbb{T}_{k}^{D}=\mathcal{P}_{k} \bmod \mathbb{Z}^{D}$, являющиеся по теореме 2.1 множествами ограниченного остатка. Тор с разбиением (4.8) будем обозначать

$$
\mathbb{T}_{\mathbf{c}, \lambda}^{D}=\mathbb{T}\left(T_{\mathbf{c}, \lambda}^{D}\right) .
$$

Из последовательности шагов (3.17), (4.7) и (4.8) складывается следующий алгоритм построения разбиений тора $\mathbb{T}_{\mathbf{c}, \lambda}^{D}$.

ОБЩАЯ СХЕМА ДЛЯ КАНОНИЧЕСКОГО РАЗБИЕНИЯ ТОРА $\mathbb{T}_{\mathbf{c}, \lambda}^{D}$ :

$$
\mathcal{C} \Longrightarrow \mathcal{C}_{\mathbf{c}} \Longrightarrow \mathcal{C}_{\mathbf{c}, \lambda} \Longrightarrow T_{\mathbf{c}, \lambda}^{D} \Longrightarrow \mathbb{T}_{\mathbf{c}, \lambda}^{D}
$$

\section{5. Отклонения и $B R$-многогранники}

5.1. Многомерная теорема Гекке. Пусть $T_{\mathbf{c}, \lambda}^{D}$ - перекладывающаяся торическая развертка (4.6) с разбиением $T_{\mathbf{c}, \lambda}^{D}=\mathcal{P}_{0} \sqcup \mathcal{P}_{1} \sqcup \cdots \sqcup \mathcal{P}_{D}$ и с заданным на ней перекладыванием $S_{v}$ (см. определение (1.5)). Указанной развертке $T_{\mathbf{c}, \lambda}^{D}$ в силу (4.9) соответствует разбиение тора $\mathbb{T}_{\mathbf{c}, \lambda}^{D}=\mathbb{T}_{0}^{D} \sqcup \mathbb{T}_{1}^{D} \sqcup \cdots \sqcup \mathbb{T}_{D}^{D}$, на котором определен сдвиг $S_{\alpha}(x) \equiv x+\alpha \bmod \mathbb{Z}^{D}$.

Введем обозначение (ср. (1.20))

$$
\beta=\frac{1}{h}(\alpha+l)
$$


при этом $h$ - произвольное натуральное число и $l \in \mathbb{Z}^{D}$ - целый вектор. Определим считающуюю функиию

$$
r_{k}\left(i ; \beta, x_{0}, \mathbb{T}_{\mathbf{c}, \lambda}^{D}\right)=\#\left\{j: S_{\beta}^{j}\left(x_{0}\right) \in \mathbb{T}_{k}^{D}, 0 \leqslant j<i\right\},
$$

где $S_{\beta}-$ сдвиг тора $S_{\beta}(x) \equiv x+\beta \bmod \mathbb{Z}^{D}$ на вектор $\beta$, определенный равенством (5.1), и $x_{0}$ - произвольная начальная точка на торе $\mathbb{T}^{D}$. Пусть вектор $\alpha=\left(\alpha_{1}, \ldots, \alpha_{D}\right)$ записан в единичном базисе $\mathbf{e}_{1}, \ldots, \mathbf{e}_{D}$ и $\alpha_{0}=1-\alpha_{1}-\cdots-\alpha_{D}$. Обозначим через

$$
\delta_{k}\left(i ; \beta, x_{0}, \mathbb{T}_{\mathbf{c}, \lambda}^{D}\right)=r_{k}\left(i ; \beta, x_{0}, \mathbb{T}_{\mathbf{c}, \lambda}^{D}\right)-i \alpha_{k} \quad \text { для } \quad k=0,1, \ldots, D
$$

отклонение распределения точек орбиты $\operatorname{Orb}\left(S_{\beta}, x_{0}\right)=\left\{S_{\beta}^{j}\left(x_{0}\right), j=0,1,2, \ldots\right\}$ относительно области $\mathbb{T}_{k}^{D}$ на торе $\mathbb{T}^{D}$.

Tеорема 5.1 (многомерная теорема Гекке). 1) Пусть $\mathbf{c} \in \mathbf{C}_{\leqslant 1}$ и вектор сдвига выбран в виде $\alpha=\lambda \mathbf{c}$, где $0<\lambda \leqslant 1$ в случае $\mathbf{c} \in \mathbf{C}_{<1} u 0<\lambda<1$, если $\mathbf{c} \in \mathbf{C}_{1}$. Тогда для отклонений (5.2) выполняются неравенства

$$
\left|\delta_{k}\left(i ; \beta, x_{0}, \mathbb{T}_{\mathbf{c}, \lambda}^{D}\right)\right| \leqslant c_{k}\left(\mathcal{C}_{\mathbf{c}}\right) h
$$

для всех $i=0,1,2, \ldots$. Константы $c_{k}\left(\mathcal{C}_{\mathbf{c}}\right)$ вычисляются по формуле

$$
c_{k}\left(\mathcal{C}_{\mathbf{c}}\right)=\max _{v \in V\left(\mathcal{C}_{\mathbf{c}}\right)} \mathbf{e}_{k} \cdot v-\min _{v \in V\left(\mathcal{C}_{\mathbf{c}}\right)} \mathbf{e}_{k} \cdot v
$$

где $\mathbf{e}_{k}-$ единичные векторы для $k=1, \ldots, D$ и $\mathbf{e}_{0}=-\mathbf{e}_{1}-\cdots-\mathbf{e}_{D}$. Здесъ через $V\left(\mathcal{C}_{\mathbf{c}}\right)$ обозначено множество вершин вытянутого параллелоэдра $\mathcal{C}_{c}$. Таким образом, константв $c_{k}\left(\mathcal{C}_{\mathbf{c}}\right)$ в неравенстве (5.3) не зависят от выбора множителя $h$ в определении (5.1) вектора сдвига $\beta$.

2) Если вектор $\mathbf{c}=\left(c_{1}, \ldots, c_{D}\right)$ записан в единичном базисе $\mathbf{e}_{1}, \ldots, \mathbf{e}_{D}$, то константь $c_{k}\left(\mathcal{C}_{\mathbf{c}}\right)$ въчисляются по формулам

$$
c_{k}\left(\mathcal{C}_{\mathbf{c}}\right)=1+(D-1) c_{k}
$$

$\partial л я k=1, \ldots, D u$

$$
c_{k}\left(\mathcal{C}_{\mathbf{c}}\right)=1+(D-1)(1-\sigma(\mathbf{c}))
$$

$\partial л я k=0$.

3) Для констант $c_{k}\left(\mathcal{C}_{\mathbf{c}}\right)$ из $(5.5),(5.6)$ выполняется общее неравенство

$$
c_{k}\left(\mathcal{C}_{\mathbf{c}}\right) \leqslant D \quad \text { для любого } \mathbf{c} \in \mathbf{C}_{\leqslant 1} .
$$

ДокАзАТЕЛЬство. Неравенство (5.3) и формула (5.4) являются частными случаями неравенства (2.14) из теоремы 2.1 и формулы (2.12). Константы (5.5) и (5.6) вычислены в [10].

5.2. $\boldsymbol{B} \boldsymbol{R}$-многогранники. Напомним, что согласно (3.6) параллелоэдр $\mathcal{C}$ натянут на векторы $\mathbf{e}_{1, \mathbf{c}}=\mathbf{e}_{1}-\mathbf{c}, \ldots, \mathbf{e}_{D, \mathbf{c}}=\mathbf{e}_{D}-\mathbf{c}$, а вытянутый параллелоэдр $\mathcal{C}_{\mathbf{c}}$ по определению (3.9) имеет вид

$$
\mathcal{C}_{\mathbf{c}}=\mathcal{C}+\mathbf{c} \cdot I
$$

Отсюда и из определения (3.17) разбиения $\mathcal{C}_{\mathbf{c}, \lambda}$ параллелоэдра $\mathcal{C}_{\mathbf{c}}$ следует, что замкнутые многогранники $\overline{\mathcal{P}}_{k}$ с номерами $k=1, \ldots, D$ допускают простое описание

$$
\overline{\mathcal{P}}_{k}=\bar{P}_{k}+\omega \text {. }
$$

Здесь $\bar{P}_{k} \subset \mathbb{R}^{D}$ обозначает замкнутый параллелепипед, натянутый на векторы

$$
\alpha, \quad \mathbf{c}-\mathbf{e}_{k^{\prime}}, \quad k^{\prime}=1, \ldots, D, \quad k^{\prime} \neq k,
$$


где $\mathbf{c}=\lambda \alpha$ и $\mathbf{e}_{k^{\prime}}-$ единичные векторы. Точка

$$
\omega=\mathbf{e}_{0}-(D-1) \mathbf{c}-\alpha,
$$

где $\mathbf{e}_{0}=(1, \ldots, 1)$, является общей для всех могогранников $\overline{\mathcal{P}}_{k}$ для $k=0,1, \ldots, D$. При этом в случае $k=0$ многогранник $\bar{P}_{0}$ также задается равенством $(5.8)$, в котором полагаем $\omega=0$ и $\overline{\mathcal{P}}_{0}$ - вытянутый параллелоэдр вида (см. (3.9))

$$
\overline{\mathcal{P}}_{0}=\operatorname{Str}_{\mathbf{c}-\alpha}(\mathcal{C}) \text {. }
$$

Если $\alpha=\left(\alpha_{1}, \ldots, \alpha_{D}\right)$ и $\alpha \in \mathbf{C}_{<1}$, то из определения (5.8) следует (вычисления приведены в [9])

$$
\operatorname{vol} \bar{P}_{k}=\alpha_{k} .
$$

В (4.5) были определены незамкнутые многогранники $\mathcal{P}_{k}$, содержащие все внутренние и некоторые граничные точки многогранника $\mathcal{P}_{k}^{c}$. Используя $\mathcal{P}_{k}$, определим соответсвующие незамкнутые многогранники $P_{k}$ равенством (ср. (5.8))

$$
\mathcal{P}_{k}=P_{k}+\omega \quad \text { для } \quad k=0,1, \ldots, D .
$$

Множество $X \subset \mathbb{R}^{D}$ назовем $\mathbb{Z}^{D}$-различимым, если оно удовлетворяет свойству

$$
x-y \in \mathbb{Z}^{D} \Longrightarrow x=y \quad \text { для всех } \quad x, y \in X .
$$

Из определения (5.13) следует, что все многогранники $P_{k}$ являются $\mathbb{Z}^{D}$-различимыми, так как согласно теореме 4.1 все их сдвиги в совокупности образуют торическую развертку $T_{\mathbf{c}, \lambda}^{D}$. Теперь мы хотим ввести в обозначение многогранников $P_{k}$ все независимые определяющие их параметры. Пусть даны произвольный вектор сдвига $\beta \in \mathbb{R}^{D}$ и число $h=1,2,3, \ldots$ такие, что существует вектор $\alpha$, удовлетворяющий условиям

$$
\alpha \in \mathbf{C}_{<1}, \quad \alpha \equiv h \beta \bmod \mathbb{Z}^{D} .
$$

Обозначим через

$$
P_{k}=P_{\beta, h, \lambda, k}
$$

многогранники, определенные равенствами (5.13) для векторов $\alpha$ из (5.15), и с $=\lambda \alpha$, где $\sigma(\alpha)<\lambda \leqslant 1$.

Для сдвига $S_{\beta}$ тора $\mathbb{T}^{D}$ и многогранника $P_{k}$ зададим считающую функцию

$$
r\left(i ; \beta, x_{0}, P_{k}\right)=\#\left\{j: S_{\beta}^{j}\left(x_{0}\right) \in P_{k} \bmod \mathbb{Z}^{D}, 0 \leqslant j<i\right\},
$$

где $x_{0}$ - произвольная начальная точка на торе $\mathbb{T}^{D}$. Также зададим отклонение

$$
\delta\left(i ; \beta, x_{0}, P_{k}\right)=r\left(i ; \beta, x_{0}, P_{k}\right)-i \alpha_{k}
$$

распределения точек орбиты $\operatorname{Orb}\left(S_{\beta}, x_{0}\right)=\left\{S_{\beta}^{j}\left(x_{0}\right), j=0,1,2, \ldots\right\}$ относительно области $\mathbb{P}_{k}^{D} \equiv P_{k} \bmod \mathbb{Z}^{D}$ на торе $\mathbb{T}^{D}$.

Теорема 5.2. Пусть данъ вектор сдвига $\beta \in \mathbb{R}^{D}$ и $h=1,2,3, \ldots$, удовлетворяющие условиям (5.15), с $=\lambda \alpha$, где $\sigma(\alpha)<\lambda \leqslant 1$, и пусть $P_{k}=P_{\beta, h, \lambda, k}$ - отвечающие этим параметрам многогранники (5.16). При этих условиях имеют место следующие утверждения.

1) Для отклонений (5.17) выполняются неравенства

$$
\left|\delta\left(i ; \beta, x_{0}, P_{k}\right)\right| \leqslant h \gamma_{k}
$$

с константами

$$
\gamma_{k}=1+(D-1) c_{k}
$$


$\partial л я k=1, \ldots, D u$

$$
\gamma_{k}=1+(D-1)(1-\sigma(\mathbf{c}))
$$

для $k=0$, где $\mathbf{c}=\left(c_{1}, \ldots, c_{D}\right)$.

2) Также для отклонений выполняется общее неравенство

$$
\left|\delta\left(i ; \beta, x_{0}, P_{k}\right)\right| \leqslant h D .
$$

ДокАЗАТЕЛЬСтво. Пусть точка $\omega$ определена в $(5.10)$, и пусть $\omega=0$ в случае $k=0$. Тогда имеет место тождество

$$
\delta\left(i ; \beta, x_{0}, P_{k}\right)=\delta_{k}\left(i ; \beta, x_{0}+\omega, \mathbb{T}_{\mathbf{c}, \lambda}^{D}\right) .
$$

Поэтому утверждение 1) непосредственно вытекает из многомерной теоремы Гекке 5.1, а неравенства (5.21) следуют из (5.18)-(5.20), если учесть, что вектор с $=\lambda \alpha$ для $\alpha \in \mathbf{C}_{<1}$ (см. (3.12)) имеет координаты $0<c_{k}<1$.

Как уже отмечалось, многогранники $P_{k}$, определенные в $(5.13)$, являются $\mathbb{Z}^{D}$-различимыми, а из теоремы 5.2 следует, что относительно сдвига $S_{\beta}$ многогранники $P_{k}$, если их рассматривать как области $\mathbb{P}_{k}^{D} \equiv P_{k} \bmod \mathbb{Z}^{D}$ на торе $\mathbb{T}^{D}$, являются множествами ограниченного остатка. Многогранники, обладающие указанными свойствами, естественно назвать многогранниками ограниченного остатка или, кратко, BR-многогранниками.

Для произвольной размерности $D$ многогранники $P_{k}$ с номерами $k=1, \ldots, D$ (по своей конструкции - параллелепипеды (5.9)) представляют собой простейший вид $B R$-многогранников. Многогранник $P_{0}$ также может быть параллелепипедом в исключительном случае вектора $\mathbf{c}=\lambda \alpha$, когда $\lambda=1$. Если же $\lambda<1$, то $P_{0}$ - параллелоэдр с числом вершин $\# V\left(P_{k}\right)=$ $2^{D+1}-2$.

Наконец заметим, что приведенное во введении неравенство (0.5) вытекает из неравенства (5.21).

\section{Список литературы}

[1] E. Hecke, "Über analytische Funktionen und die Verteilung von Zahlen mod. eins", Hamb. Abh., 1:1 (1922), 54-76.

[2] I. Oren, "Admissible functions with multiple discontinuities", Proceedings of the Special Seminar on Topology, Vol. I, Univ. Nac. Autónoma México, Mexico City, 1981, 217-230.

[3] R. Szüsz, "Über die Verteilung der Vielfachen einer komplexen Zahl nach dem Modul des Einheitsquadrats", Acta Math. Acad. Sci. Hungar., 5:1-2 (1954), 35-39.

[4] P. Liardet, "Regularities of distribution", Compositio Math., 61:3 (1987), 267-293.

[5] G. Rauzy, "Ensembles à restes bornés", Seminaire de theorie des nombres de Bordeaux 1983/1984, Exp. No. 24, Univ. Bordeaux I, Talence, 1984.

[6] S. Ferenczi, "Bounded remainder sets", Acta Arith., 61:4 (1992), 319-326.

[7] В. Г. Журавлёв, "Разбиения Рози и множества ограниченного остатка на торе”, Труды по теории чисел, Зап. научн. сем. ПОМИ, 322, ПОМИ, СПб., 2005, 83-106.

[8] В. Г. Журавлёв, "Многомерная теорема Гекке о распределении дробных частей”, Алгебра и анализ, 24:1 (2012), 95-130.

[9] В. Г. Журавлёв, "Перекладывающиеся торические развертки и множества ограниченного остатка", Аналитическая теория чисел и теория функиий. 26, Зап. научн. сем. ПОМИ, 392, ПОМИ, СПб., 2011, 95-145.

[10] В. Г. Журавлёв, "Модули торических разбиений на множества ограниченного остатка и сбалансированные слова", Алгебра и анализ, 24:4 (2012), 97-136.

[11] Г. Ф. Вороной, Собрание сочинений, В 3-х томах, Т. 2, АН УССР, Киев, 1952.

[12] H. Weyl, "Über die Gleichverteilung von Zahlen mod. Eins", Math. Ann., 77:3 (1916), 313-352. 